\title{
Coupled Dispersionless and Generalized Heisenberg Ferromagnet Equations with Self-Consistent Sources: Geometry and Equivalence
}

\author{
Guldana Bekova* Gulgassyl Nugmanovał Gaukhar Shaikhova \\ Kuralay Yesmakhanova ${ }^{\S}$ and Ratbay Myrzakulov \\ Eurasian International Center for Theoretical Physics and \\ Department of General \& Theoretical Physics, \\ Eurasian National University, Astana, 010008, Kazakhstan
}

January 17, 2019

\begin{abstract}
We propose a new integrable coupled dispersionless equation with self-consistent sources (CDESCS). We obtain the Lax pair and the equivalent generalized Heisenberg ferromagnet equation (GHFE), demonstrating its integrability. Specifically, we explore the geometry of these equations. Last, we consider the relation between the motion of curves/surfaces and the CDESCS and the GHFE.
\end{abstract}

\section{Introduction}

The study of integrable systems or solvable nonlinear differential equations (NDE) is an active area of research since the discovery of the inverse scattering method. These equations are in a sense universal since they show up in many areas of physics and mathematics. As integrable systems we understand those which have infinite hierarchy of symmetries and conservation laws. In the theory of solvable nonlinear differential equations, one of the most important issues is a systematic method for construction of integrable systems. For integrable systems there exist several parallel schemes of construction.

\footnotetext{
*Email: bekovaguldana@gmail.com

†Email: nugmanovagn@gmail.com

†Email: g.shaikhova@gmail.com

$\S$ Email: kryesmakhanova@gmail.com

『Email: rmyrzakulov@gmail.com
} 
Besides the integrable NDEs, there is another important class of integrable partial differential equations: the so-called integrable equations of hydrodynamic type, often called dispersionless equations [1]-[7]. In some cases, these equations are the dispersionless (or semiclassical) limits of integrable soliton systems, or by construction, such as the system of hydrodynamic type. They often arise in various problems of physics and mathematics, for this reason, they are intensively studied in the recent years. Some of these equations are integrable in the Hamiltonian sense. Integrable dispersionless equations are equivalent to the commutation condition of vector fields Lax pairs, so that they can be in an arbitrary number of dimensions. For example, in [1]-[3], a new systematic method was introduced for construction of dispersionless systems in $3+1$ dimensions using nonisospectral Lax pairs that involve contact vector fields.

In this paper we deal with the so-called Konno-Oono equation (KOE) [8]

$$
\begin{aligned}
q_{x t}-\rho q & =0 \\
\rho_{x}+0.5\left(q^{2}\right)_{t} & =0 .
\end{aligned}
$$

The KOE (11) is also known as the coupled dispersionless equation (CDE). Later, Konno and Kakuhata proposed a generalization of this equation in the following form [9]

$$
\begin{aligned}
q_{x t}-\rho q & =0, \\
r_{x t}-\rho r & =0, \\
\rho_{x}+0.5(r q)_{t} & =0
\end{aligned}
$$

which is called the generalized Konno-Oono equation (GKOE) or the generalized CDE (GCDE). Its Lax representation (LR) can be expressed as

$$
\Psi_{x}=U_{1} \Psi, \quad \Phi_{t}=V_{1} \Psi
$$

where

$$
V_{1}=-i \lambda\left(\begin{array}{cc}
\rho & q_{t} \\
r_{t} & -\rho
\end{array}\right), \quad U_{1}=\left(\begin{array}{cc}
\frac{i}{4 \lambda} & -0.5 q \\
0.5 r & -\frac{i}{4 \lambda}
\end{array}\right) .
$$

It were pointed that the KOE (11) is gauge equivalent to the sine-Gordon equation, whereas its complex version that corresponds to the GKOE (3)-(5) with the reduction $r=\bar{q}$ is gauge equivalent to the Pohlmeyer-Lund-Regge equation [10]. It is interesting to note that there exist other gauge and geometrical equivalent equations to the GKOE (3) -(5) and to its reductions. These equivalent equations are some kind of generalizations of the famous Heisenberg ferromagnet equations (HFE) [61]-662]

$$
i A_{t}=\frac{1}{2}\left[A, A_{x x}\right]
$$

and which are the subject of this paper. 
The integrable systems with self-consistent sources (ISSCS) have attracted some attention (see, for example, [1]-[14]). The ISSCS can be solved by the inverse scattering transform (IST) method, and N-soliton solutions of some ISSCSs were obtained [1]-[15].

The present paper is organized as follows. In Sec. 2, we present the M-XIV equation in the matrix and the vector form. Specifically, we employ a Lax representation of this equation. In Sec. 3, we demonstrate the geometric representation of the M-XIV equation. In particular, we show that the M-XIV equation and the coupled dispersionless equation with self-consistent equation are closely related to each other. In the following Sec. 4 we establish the gauge equivalence between the M-XIV and M-XXXII equations. In Sec. 5, we analyze several reductions of the M-XXXII equation in detail and present their Lax representations. The scale transformations of the M-XXXII equation is given in Sec. 6. Integrable surface induced by the M-XIV equation is presented in Sec. 7. The corresponding surface equation is the M-XXXI equation. Section 8 is devoted to concluding remarks.

\section{Myrzakulov-XIV equation}

In this section, we present the different forms of the Myrzakulov-XIV (M-XIV) equation as well as its Lax representation, reduction and simplest conservation law.

\subsection{Equation}

\subsubsection{The matrix M-XIV equation}

Consider the following M-XIV equation

$$
\begin{aligned}
i A_{t} & =i(f A)_{x}+\frac{1}{4 \alpha}\left[A, A_{t}\right]_{x}+\frac{1}{\alpha}[A, W], \\
W_{x} & =i(\alpha-\omega)[A, W]
\end{aligned}
$$

or

$$
\begin{aligned}
i A_{t} & =\text { if } A_{x}+\frac{1}{4 \alpha}\left[A, A_{t x}\right]+\frac{1}{\alpha}[A, W], \\
f_{x} & =\frac{1}{4 i \alpha} \operatorname{tr}\left(A\left[A_{t}, A_{x}\right]\right), \\
W_{x} & =i(\alpha-\omega)[A, W] .
\end{aligned}
$$

Here

$$
\begin{aligned}
A & =\left(\begin{array}{cc}
A_{3} & \sigma A^{-} \\
A^{+} & -A_{3}
\end{array}\right), \quad A^{2}=I, \quad A^{ \pm}=A_{1} \pm i A_{2}, \quad A_{1}^{2}+A_{2}^{2}+A_{3}^{2}=1 \\
W & =\left(\begin{array}{cc}
W_{3} & \sigma W^{-} \\
W^{+} & -W_{3}
\end{array}\right), \quad W^{2}=I, \quad W^{ \pm}=W_{1} \pm i W_{2}, \quad W_{1}^{2}+W_{2}^{2}+W_{3}^{2}=1
\end{aligned}
$$




\subsubsection{The vector M-XIV equation}

In the vector form the M-XIV equation looks like

$$
\begin{aligned}
\mathbf{A}_{t} & =(f \mathbf{A})_{x}+\frac{1}{2 \alpha}\left(\mathbf{A} \wedge \mathbf{A}_{t}\right)_{x}+\frac{2}{\alpha} \mathbf{A} \wedge \mathbf{W}, \\
\mathbf{W}_{x} & =2(\omega-\alpha) \mathbf{A} \wedge \mathbf{W}
\end{aligned}
$$

or

$$
\begin{aligned}
\mathbf{A}_{t} & =f \mathbf{A}_{x}+\frac{1}{2 \alpha} \mathbf{A} \wedge \mathbf{A}_{x t}+\frac{2}{\alpha} \mathbf{A} \wedge \mathbf{W}, \\
f_{x} & =\frac{1}{2 \alpha} \mathbf{A} \cdot\left(\mathbf{A}_{t} \wedge \mathbf{A}_{x}\right) \\
\mathbf{W}_{x} & =2(\omega-\alpha) \mathbf{A} \wedge \mathbf{W}
\end{aligned}
$$

where

$$
\begin{aligned}
\mathbf{A} & =\left(A_{1}, A_{2}, A_{3}\right), \quad \mathbf{A}^{2}=1, \quad f=\frac{1}{\alpha^{2}} a+\frac{1}{\alpha(\alpha-\omega)} \eta, \\
\mathbf{W} & =\left(W_{1}, W_{2}, W_{3}\right), \quad W_{1}^{2}+W_{2}^{2}+W_{3}^{2}=\text { const }=1, \quad \mathbf{A} \cdot \mathbf{W}_{x}=0 .
\end{aligned}
$$

\subsubsection{The M-XIV equation as the equation with self-consistent sources}

We can rewrite the M-XIV equation as the equation with self-consistent sources. Let us we introduce the following representation for the components of the matrix function $W$ :

$$
\begin{aligned}
& W_{1}=\phi_{1} \bar{\phi}_{2}+\bar{\phi}_{1} \phi_{2}, \\
& W_{2}=i\left(\phi_{1} \bar{\phi}_{2}-\bar{\phi}_{1} \phi_{2}\right), \\
& W_{3}=\left|\phi_{1}\right|^{2}-\left|\phi_{2}\right|^{2}
\end{aligned}
$$

or

$$
\begin{aligned}
W^{+} & =2 \bar{\phi}_{1} \phi_{2}, \\
W^{-} & =2 \phi_{1} \bar{\phi}_{2}, \\
W_{3} & =\left|\phi_{1}\right|^{2}-\left|\phi_{2}\right|^{2},
\end{aligned}
$$

so that the matrix $W$ is given by

$$
W=\left(\begin{array}{cc}
W_{3} & W^{-} \\
W^{+} & -W_{3}
\end{array}\right)=\left(\begin{array}{cc}
\left|\phi_{1}\right|^{2}-\left|\phi_{2}\right|^{2} & 2 \phi_{1} \bar{\phi}_{2} \\
2 \bar{\phi}_{1} \phi_{2} & \left|\phi_{2}\right|^{2}-\left|\phi_{1}\right|^{2}
\end{array}\right) .
$$

Here $\phi_{j}$ obey the following set of equations

$$
\begin{aligned}
& \phi_{1 x}=-i \zeta\left(A_{3} \phi_{1}+A^{-} \phi_{2}\right), \\
& \phi_{2 x}=-i \zeta\left(A^{+} \phi_{1}-A_{3} \phi_{2}\right),
\end{aligned}
$$


where $\zeta=\omega-\alpha$. Thus the M-XIV equation (as the equation with self-consistent sources) can be written as

$$
\begin{aligned}
i A_{t} & =i f A_{x}+\frac{1}{4 \alpha}\left[A, A_{t x}\right]+F, \\
f_{x} & =\frac{1}{4 i \alpha} \operatorname{tr}\left(A\left[A_{t}, A_{x}\right]\right), \\
\phi_{1 x} & =-i \zeta\left(A_{3} \phi_{1}+A^{-} \phi_{2}\right), \\
\phi_{2 x} & =-i \zeta\left(A^{+} \phi_{1}-A_{3} \phi_{2}\right),
\end{aligned}
$$

where

$$
F=\frac{2}{\alpha}\left(\begin{array}{cc}
A^{-} \bar{\phi}_{1} \phi_{2}-A^{+} \phi_{1} \bar{\phi}_{2} & 2 A_{3} \phi_{1} \bar{\phi}_{2}-A^{-}\left(\left|\phi_{1}\right|^{2}-\left|\phi_{2}\right|^{2}\right) \\
A^{+}\left(\left|\phi_{1}\right|^{2}-\left|\phi_{2}\right|^{2}\right)-2 A_{3} \bar{\phi}_{1} \phi_{2} & A^{+} \phi_{1} \bar{\phi}_{2}-A^{-} \bar{\phi}_{1} \phi_{2}
\end{array}\right) .
$$

\subsection{Lax representation}

The M-XIV equation (9)-(10) is integrable. Its LR reads as

$$
\begin{aligned}
& \Phi_{x}=U_{2} \Phi, \\
& \Phi_{t}=V_{2} \Phi
\end{aligned}
$$

where

$$
\begin{aligned}
& U_{2}=-i(\lambda-\alpha) A \\
& V_{2}=-\frac{i(\lambda-\alpha)}{\alpha \lambda}\left(\alpha^{2} f A-\frac{i \alpha}{4}\left[A, A_{t}\right]-\frac{\alpha}{\alpha-\omega} W\right)-\frac{i(\lambda-\alpha)}{(\alpha-\omega)(\lambda-\omega)} W .
\end{aligned}
$$

\subsection{Reduction}

Let $W=0$. Then the M-XIV equation takes the form

$$
i A_{t}=i(f A)_{x}+\frac{1}{4 \alpha}\left[A, A_{t}\right]_{x}
$$

or

$$
\begin{aligned}
i A_{t} & =i f A_{x}+\frac{1}{4 \alpha}\left[A, A_{t x}\right], \\
f_{x} & =\frac{1}{4 i \alpha} \operatorname{tr}\left(A\left[A_{t}, A_{x}\right]\right),
\end{aligned}
$$

and which is the so-called M-XIII equation [94]. 


\subsection{Conservation laws}

As the integrable system, the M-XIV equation admits the infinity number of conservation

laws. The simplest one that we can get, for example, from Eqs. (18)-(20) has the following form

$$
\left(\mathbf{A}_{x}^{2}\right)_{t}+\left(8 \mathbf{A} \cdot \mathbf{W}+8 \alpha^{2} f\right)_{x}=0
$$

In $\mathbf{W}=0$ case, this equation takes the form

$$
\left(\mathbf{A}_{x}^{2}\right)_{t}+8 \alpha^{2} f_{x}=0
$$

so that

$$
\left(\mathbf{A}_{x}^{2}\right)_{t}=4 \alpha \mathbf{A} \cdot\left(\mathbf{A}_{x} \wedge \mathbf{A}_{t}\right)
$$

\section{Integrable motion of space curves. The equation Lakshmanan (geometrical) equivalent to the M- XIV equation}

In this section, we establish the link between the M-XIV equation and the motion of space curves. Then using this link we can find the Lakshmanan (geometrical) equivalent counterpart of the M-XIV equation. Consider a family of smooth space curve in $R^{3}$ which we define as

$$
\gamma(x, t):[0, X] \times[0, T] \rightarrow R^{3}
$$

where $x$ is the arc length of the curve at each time $t$. In this case, the unit tangent vector $\mathbf{e}_{1}$, principal normal vector $\mathbf{e}_{2}$ and binormal vector $\mathbf{e}_{3}$ are defined as

$$
\mathbf{e}_{1}=\gamma_{x}, \quad \mathbf{e}_{2}=\frac{\gamma_{x x}}{\left|\gamma_{x x}\right|}, \quad \mathbf{e}_{3}=\mathbf{e}_{1} \wedge \mathbf{e}_{2},
$$

respectivily. The corresponding Frenet-Serret equation reads as

$$
\left(\begin{array}{l}
\mathbf{e}_{1} \\
\mathbf{e}_{2} \\
\mathbf{e}_{3}
\end{array}\right)_{x}=C\left(\begin{array}{l}
\mathbf{e}_{1} \\
\mathbf{e}_{2} \\
\mathbf{e}_{3}
\end{array}\right)=\left(\begin{array}{ccc}
0 & \kappa_{1} & \kappa_{2} \\
-\kappa_{1} & 0 & \tau \\
-\kappa_{2} & -\tau & 0
\end{array}\right)\left(\begin{array}{l}
\mathbf{e}_{1} \\
\mathbf{e}_{2} \\
\mathbf{e}_{3}
\end{array}\right)
$$

where $\tau, \kappa_{1}$ and $\kappa_{2}$ are torsion, geodesic curvature and normal curvature of the curve, respectively. 
It is well-known that between some integrable systems there take place the geometrical (Lakshmanan) and gauge equivalences. The Frenet-Serret equation and the general temporal evolution equation are given by

$$
\left(\begin{array}{l}
\mathbf{e}_{1} \\
\mathbf{e}_{2} \\
\mathbf{e}_{3}
\end{array}\right)_{x}=C\left(\begin{array}{l}
\mathbf{e}_{1} \\
\mathbf{e}_{2} \\
\mathbf{e}_{3}
\end{array}\right), \quad\left(\begin{array}{l}
\mathbf{e}_{1} \\
\mathbf{e}_{2} \\
\mathbf{e}_{3}
\end{array}\right)_{t}=G\left(\begin{array}{l}
\mathbf{e}_{1} \\
\mathbf{e}_{2} \\
\mathbf{e}_{3}
\end{array}\right),
$$

where

$$
C=\left(\begin{array}{ccc}
0 & \kappa_{1} & \kappa_{2} \\
-\kappa_{1} & 0 & \tau \\
-\kappa_{2} & -\tau & 0
\end{array}\right), \quad G=\left(\begin{array}{ccc}
0 & \omega_{3} & \omega_{2} \\
-\omega_{3} & 0 & \omega_{1} \\
-\omega_{2} & -\omega_{1} & 0
\end{array}\right)
$$

The compatibility condition of these equations is written as

$$
C_{t}-G_{x}+[C, G]=0
$$

or in elements

$$
\begin{aligned}
\kappa_{1 t}-\omega_{3 x}-\kappa_{2} \omega_{1}+\tau \omega_{2} & =0, \\
\kappa_{2 t}-\omega_{2 x}+\kappa_{1} \omega_{1}-\tau \omega_{3} & =0, \\
\tau_{t}-\omega_{1 x}-\kappa_{1} \omega_{2}+\kappa_{2} \omega_{3} & =0 .
\end{aligned}
$$

Our next step is the following identification

$$
\mathbf{A} \equiv \mathbf{e}_{1}
$$

and the assumption

$$
\kappa_{1}=-2 \zeta, \quad \kappa_{2}=r-q, \quad \tau=-i(r+q),
$$

where $q=-0.5\left(\kappa_{2}-i \tau\right)$ and $r=0.5\left(\kappa_{2}+i \tau\right)$ are some functions, $\zeta=$ const. Then we have

$$
\begin{aligned}
& \omega_{1}=\frac{\left.0.5 r_{t}-0.5 q_{t}-n-p\right)}{\zeta}+\frac{n+p}{\zeta-\omega}, \\
& \omega_{2}=\frac{i\left(0.5 r_{t}+0.5 q_{t}-n+p\right)}{\zeta}+\frac{n-p}{\zeta-\omega}, \\
& \omega_{3}=\frac{2 \eta}{\zeta-\omega}+\frac{2 a}{\zeta} .
\end{aligned}
$$

The Eqs.(34)-(35) give us the following equations for $q, r, \rho=4 a, n, p, \eta$ :

$$
\begin{aligned}
q_{x t}-\rho q+4 p_{x} & =0, \\
r_{x t}-\rho r-4 n_{x} & =0, \\
\rho_{x}-0.5(r q)_{t}+2(q n-r p) & =0, \\
\eta_{x}+0.5(r p-q n) & =0, \\
p_{x}+2 i \omega p+\eta q & =0, \\
n_{x}-2 i \omega n-\eta r & =0
\end{aligned}
$$


which is the so-called Myrzakulov-XXXII (M-XXXII) equation. Note that for the reductions $r=\sigma \bar{q}$ and $n=-\sigma \bar{p}$, the M-XXXII equation (44)-(49) takes the form

$$
\begin{aligned}
q_{x t}-4 a q+2 p_{x} & =0 \\
a_{x}-\sigma\left[0.5\left(|q|^{2}\right)_{t}+q \bar{p}+\bar{q} p\right] & =0 \\
\eta_{x}+\sigma(\bar{q} p+q \bar{p}) & =0 \\
p_{x}+2 i \omega p+2 \eta q & =0
\end{aligned}
$$

So, we have proved the Lakshmanan (geometrical) equivalence between the M-XIV equation (9)-(10) and the M-XXXII equation (67)-(70).

\section{On the equation gauge equivalent to the M-XIV equation}

Taking into account the results of the previous section, we expect that there exists gauge equivalence between the M-XIV equation (9)-(10) and the M-XXXII equation (44)-(49). To check it, consider the following transformation $\Phi=g \Psi$, where $\Phi$ is some solutions of the set (37)-(38) and $g=\left.\Psi\right|_{\lambda=\alpha}$. Then the function $\Psi$ obeys the following set of linear equations

$$
\begin{aligned}
\Psi_{x} & =U_{5} \Psi \\
\Phi_{t} & =V_{5} \Psi
\end{aligned}
$$

Here

$$
U_{5}=-i \lambda \sigma_{3}+Q, \quad V_{5}=\frac{i}{\lambda} F+\frac{i}{\lambda-\omega} G
$$

where

$$
Q=\left(\begin{array}{cc}
0 & q \\
\sigma \bar{q} & 0
\end{array}\right), \quad F=\left(\begin{array}{cc}
a & -0.5 q_{t}-p \\
0.5 \sigma\left(\bar{q}_{t}+\bar{p}\right) & -a
\end{array}\right), \quad G=\left(\begin{array}{cc}
\eta & p \\
-\sigma \bar{p} & -\eta
\end{array}\right) .
$$

It can be easily shown that the compatibility condition $U_{5 t}-V_{5 x}+\left[U_{5}, V_{5}\right]=0$ gives the M-XXXII equation (61)-(66).

\section{The M-XXXII equation}

For our convenience, here we collect the main formulas of the M-XXXII equation (61)-(66). 


\subsection{Equation}

The M-XXXII equation (44)-(49) reads as

$$
\begin{aligned}
q_{x t}-4 a q+2 p_{x} & =0, \\
r_{x t}-4 a r-2 n_{x} & =0, \\
a_{x}-0.5(r q)_{t}+q n-r p & =0, \\
\eta_{x}+r p-q n & =0, \\
p_{x}+2 i \omega p+2 \eta q & =0, \\
n_{x}-2 i \omega n-2 \eta r & =0 .
\end{aligned}
$$

\subsection{Lax representation}

The M-XXXII equation is integrable and its LR is

$$
\begin{aligned}
& \Psi_{x}=U_{3} \Psi, \\
& \Psi_{t}=V_{3} \Psi .
\end{aligned}
$$

Here

$$
U_{3}=-i \lambda \sigma_{3}+Q, \quad V_{3}=\frac{i}{\lambda} F+\frac{i}{\lambda-\omega} G
$$

where

$$
Q=\left(\begin{array}{ll}
0 & q \\
r & 0
\end{array}\right), \quad F=\left(\begin{array}{cc}
a & -0.5 q_{t}-p \\
0.5 r_{t}-n & -a
\end{array}\right), \quad G=\left(\begin{array}{cc}
\eta & p \\
n & -\eta
\end{array}\right) .
$$

Note that from Eqs. (78)-(80) we get the following important formula

$$
\eta^{2}+n p=\text { const }
$$

or, for simplicity,

$$
\eta^{2}+n p=1 .
$$

\subsection{Reductions}

\subsubsection{Case 1: $r=\sigma q$}

First we consider the particular case when

$$
r=\sigma q
$$


Then we have

$$
\begin{aligned}
q_{x t}-4 a q+2 p_{x} & =0, \\
\eta_{x}+2 \sigma q p & =0, \\
p_{x}+2 i \omega p+2 \eta q & =0,
\end{aligned}
$$

where $n=-\sigma p$ and $\omega=\bar{\omega}$. This equation is also integrable and its LR is

$$
\begin{aligned}
& \Psi_{x}=U_{4} \Psi, \\
& \Psi_{t}=V_{4} \Psi .
\end{aligned}
$$

Here

$$
U_{4}=-\lambda \sigma_{3}+Q, \quad V_{4}=\frac{i}{\lambda} F+\frac{i}{\lambda-\omega} G
$$

where

$$
Q=\left(\begin{array}{cc}
0 & q \\
\sigma q & 0
\end{array}\right), \quad F=\left(\begin{array}{cc}
a & -0.5 q_{t}-p \\
0.5 \sigma q_{t}+\sigma p & -a
\end{array}\right), \quad G=\left(\begin{array}{cc}
\eta & p \\
\sigma p & -\eta
\end{array}\right) .
$$

\subsubsection{Case 2: $r=\sigma \bar{q}$}

In this case that is when

$$
r=\sigma \bar{q}, \quad n=-\sigma \bar{p},
$$

we get the following set of equations

$$
\begin{aligned}
q_{x t}-4 a q+2 p_{x} & =0 \\
a_{x}-\sigma\left[0.5\left(|q|^{2}\right)_{t}+q \bar{p}+\bar{q} p\right] & =0 \\
\eta_{x}+\sigma(\bar{q} p+q \bar{p}) & =0 \\
p_{x}+2 i \omega p+2 \eta q & =0 .
\end{aligned}
$$

This reduction of the M-XXXII equation is integrable with the following LR

$$
\begin{aligned}
\Psi_{x} & =U_{5} \Psi, \\
\Phi_{t} & =V_{5} \Psi .
\end{aligned}
$$

Here

$$
U_{5}=-\lambda \sigma_{3}+Q, \quad V_{5}=\frac{i}{\lambda} F+\frac{i}{\lambda-\omega} G
$$

where

$$
Q=\left(\begin{array}{cc}
0 & q \\
\sigma \bar{q} & 0
\end{array}\right), \quad F=\left(\begin{array}{cc}
a & -0.5 q_{t}-p \\
0.5 \sigma\left(\bar{q}_{t}+\bar{p}\right) & -a
\end{array}\right), \quad G=\left(\begin{array}{cc}
\eta & p \\
-\sigma \bar{p} & -\eta
\end{array}\right) .
$$




\subsubsection{Case 3: $p=n=\eta=0$}

In this particular case when

$$
p=n=\eta=0,
$$

we have

$$
\begin{aligned}
q_{x t}-4 a q & =0, \\
r_{x t}-4 a r & =0, \\
a_{x}-0.5(r q)_{t} & =0,
\end{aligned}
$$

which is, in fact, the GKOE (33)-(5) .

\subsection{The M-XXXII equation as the equation with self-consistent sources}

We can rewrite the M-XXXII equation as the equation with self-consistent sources. Let us consider the following representation for the functions $p, n$ and $\eta$ :

$$
p=2 \psi_{1} \bar{\psi}_{2}, \quad n=-2 \sigma \bar{\psi}_{1} \psi_{2}, \quad \eta=\left|\psi_{1}\right|^{2}+\sigma\left|\psi_{2}\right|^{2},
$$

where $\left.\psi_{1}\right|^{2}-\sigma\left|\psi_{2}\right|^{2}=$ const and $\Psi=\left(\psi_{1}, \psi_{2}\right)^{T}$ satisfy the equations (81)-(82). Then the M-XXXII equation takes the form

$$
\begin{aligned}
q_{x t}-4 a q+4\left(\psi_{1} \bar{\psi}_{2}\right)_{x} & =0 \\
a_{x}-0.5 \sigma\left(|q|^{2}\right)_{t}-2 \sigma\left(q \bar{\psi}_{1} \psi_{2}+\bar{q} \psi_{1} \bar{\psi}_{2}\right) & =0 \\
\psi_{1 x}+i \xi \psi_{1}-q \psi_{2} & =0 \\
\psi_{2 x}-i \xi \psi_{2}-\sigma \bar{q} \psi_{1} & =0
\end{aligned}
$$

or

$$
\begin{aligned}
q_{x t}-4 a q-8 i \xi \psi_{1} \bar{\psi}_{2}+4 q\left(\sigma\left|\psi_{1}\right|^{2}+\left|\psi_{2}\right|^{2}\right) & =0 \\
a_{x}-0.5 \sigma\left(|q|^{2}\right)_{t}-2 \sigma\left(q \bar{\psi}_{1} \psi_{2}+\bar{q} \psi_{1} \bar{\psi}_{2}\right) & =0 \\
\psi_{1 x}+i \xi \psi_{1}-q \psi_{2} & =0 \\
\psi_{2 x}-i \xi \psi_{2}-\sigma \bar{q} \psi_{1} & =0 .
\end{aligned}
$$

This is the desired form of the M-XXXII equation written as the equation with selfconsistent sources. 


\section{Scale transformation}

Consider the following scale transformation

$$
a \rightarrow 0.25 \rho, \quad(q, r) \rightarrow 0.5(q, r)
$$

Under this transformation, the M-XXXII equation (44)-(49) becomes

$$
\begin{aligned}
q_{x t}-\rho q+4 p_{x} & =0 \\
r_{x t}-\rho r-4 n_{x} & =0 \\
\rho_{x}-0.5(r q)_{t}+2(q n-r p) & =0 \\
\eta_{x}+0.5(r p-q n) & =0 \\
p_{x}+2 i \omega p+\eta q & =0 \\
n_{x}-2 i \omega n-\eta r & =0 .
\end{aligned}
$$

When $p=n=\eta=0$, it looks like

$$
\begin{aligned}
q_{x t}-\rho q & =0, \\
r_{x t}-\rho r & =0, \\
\rho_{x}-0.5(r q)_{t} & =0,
\end{aligned}
$$

which is a more standard form of the GKOE (3) $-(\sqrt[51]{1})$ with $(r) \rightarrow(-r)$.

\section{Integrable surface induced by the M-XIV equation}

In this section, we present a surface which is induced by the M-XIV equation. Let us consider a surface in three-dimensional Euclidean space $R^{3}$ that is parametrized by position vector $\mathbf{r}(x, t)$ of the surface. To construct the surface, let us do the following identification

$$
\mathbf{A} \equiv \mathbf{r}_{x}
$$

where $\mathbf{r}_{x}^{2}=1$. After that from the vector M-XIV equation (16)-(17) we get the following Myrzakulov-XXXI (M-XXXI) equation

$$
\begin{aligned}
\mathbf{r}_{t} & =f \mathbf{r}_{x}+\frac{1}{2 \alpha} \mathbf{r}_{x} \wedge \mathbf{r}_{x t}+\frac{1}{\alpha(\omega-\alpha)} \mathbf{W}, \\
f_{x} & =\frac{1}{2 \alpha} \mathbf{r}_{x} \cdot\left(\mathbf{r}_{x t} \wedge \mathbf{r}_{x x}\right), \\
\mathbf{W}_{x} & =2(\omega-\alpha) \mathbf{r}_{x} \wedge \mathbf{W},
\end{aligned}
$$


where $\mathbf{r}=\left(r_{1}, r_{2}, r_{3}\right)$. The M-XXXI equation (128)-(130) is integrable. Its LR looks as

$$
\begin{aligned}
& \Phi_{x}=U_{7} \Phi, \\
& \Phi_{t}=V_{7} \Phi,
\end{aligned}
$$

where

$$
\begin{aligned}
U_{7} & =-i(\lambda-\alpha) r_{x} \\
V_{7} & =-\frac{i(\lambda-\alpha)}{\alpha \lambda}\left(\alpha^{2} f r_{x}-\frac{i \alpha}{4}\left[r_{x}, r_{x t}\right]-\frac{\alpha}{\alpha-\omega} W\right)-\frac{i(\lambda-\alpha)}{(\alpha-\omega)(\lambda-\omega)} W .
\end{aligned}
$$

The compatibility condition of the equations (131)-(132) gives the following equation

$$
\begin{aligned}
i r_{t} & =i f r_{x}+\frac{1}{4 \alpha}\left[r, r_{t}\right]+\frac{i}{\alpha(\alpha-\omega)} W, \\
f_{x} & =\frac{1}{4 i \alpha} \operatorname{tr}\left(r_{x}\left[r_{x t}, r_{x x}\right]\right), \\
W_{x} & =i(\alpha-\omega)\left[r_{x}, W\right]
\end{aligned}
$$

which is the matrix form of the M-XXXI equation. Here

$$
r=\left(\begin{array}{cc}
r_{3} & r^{-} \\
r^{+} & -r_{3}
\end{array}\right), \quad r_{x}^{2}=I, \quad r_{1 x}^{2}+r_{2 x}^{2}+r_{3 x}^{2}=1, \quad r^{ \pm}=r_{1} \pm i r_{2} .
$$

In our case, the first fundamental form of the surface is given by

$$
I=d x^{2}+2\left(\mathbf{r}_{x} \cdot \mathbf{r}_{t}\right) d x d t+\mathbf{r}_{t}^{2} d t^{2},
$$

where

$$
\begin{aligned}
\mathbf{r}_{x}^{2} & =1, \\
\mathbf{r}_{x} \cdot \mathbf{r}_{t} & =f+\frac{1}{\alpha(\omega-\alpha)} \mathbf{r}_{x} \cdot \mathbf{W}, \\
\mathbf{r}_{t}^{2} & =f^{2}+\frac{1}{\alpha^{2}(\omega-\alpha)^{2}}+\frac{2 f\left(\mathbf{r}_{x} \cdot \mathbf{W}\right)}{\alpha(\omega-\alpha)}+\frac{\left|\mathbf{r}_{x} \wedge \mathbf{r}_{x t}\right|^{2}}{4 \alpha^{2}}+\frac{\left(\mathbf{r}_{x} \wedge \mathbf{r}_{x t}\right) \cdot \mathbf{W}}{\alpha^{2}(\omega-\alpha)} .
\end{aligned}
$$

Finally, we note that the M-XXXI equation (128)-(130) can be rewritten as

$$
\begin{aligned}
\mathbf{r}_{x t} & =2 \alpha \mathbf{r}_{t} \wedge \mathbf{r}_{x}+\frac{2}{\alpha-\omega} \mathbf{r}_{x} \wedge \mathbf{W}, \\
\mathbf{W}_{x} & =2(\omega-\alpha) \mathbf{r}_{x} \wedge \mathbf{W},
\end{aligned}
$$

or

$$
\begin{aligned}
\mathbf{r}_{x t} & =2 \alpha \mathbf{r}_{t} \wedge \mathbf{r}_{x}-\frac{1}{(\alpha-\omega)^{2}} \mathbf{W}_{x}, \\
\mathbf{W}_{x} & =2(\omega-\alpha) \mathbf{r}_{x} \wedge \mathbf{W} .
\end{aligned}
$$


The above obtained results are enough to define the surface in three-dimensional Euclidean space $R^{3}$ parametrized by the position vector $\mathbf{r}(x, t)$ of the surface. This surface is integrable as its equation that is the equation for $\mathbf{r}$ (128)-(130) admits LR. So we have shown that the M-XXXI equation induces the some integrable surface. Lastly, we present the reduction of the $\mathrm{M}-\mathrm{XXXI}$ equation. If $\mathbf{W}=0$, then it turns to the following equation 52

$$
\mathbf{r}_{t}=f \mathbf{r}_{x}+\frac{1}{2 \alpha} \mathbf{r}_{x} \wedge \mathbf{r}_{x t}
$$

or

$$
\mathbf{r}_{x t}=2 \alpha \mathbf{r}_{t} \wedge \mathbf{r}_{x}
$$

where

$$
f=\mathbf{r}_{t} \cdot \mathbf{r}_{x}
$$

Finally, let us present the M-XXXI equation (128)-(130) as the equation with self-consistent sources. It has the form

$$
\begin{aligned}
r_{1 t} & =f r_{1 x}+\frac{1}{2 \alpha}\left(r_{2 x} r_{3 x t}-r_{2 x t} r_{3 x}\right)+\frac{1}{\alpha(\omega-\alpha)}\left(\phi_{1} \bar{\phi}_{2}+\bar{\phi}_{1} \phi_{2}\right), \\
r_{2 t} & =f r_{2 x}+\frac{1}{2 \alpha}\left(r_{3 x} r_{1 x t}-r_{3 x t} r_{1 x}\right)+\frac{i}{\alpha(\omega-\alpha)}\left(\phi_{1} \bar{\phi}_{2}-\bar{\phi}_{1} \phi_{2}\right), \\
r_{3 t} & =f r_{3 x}+\frac{1}{2 \alpha}\left(r_{1 x} r_{2 x t}-r_{1 x t} r_{2 x}\right)+\frac{1}{\alpha(\omega-\alpha)}\left(\left|\phi_{1}\right|^{2}-\left|\phi_{2}\right|^{2}\right), \\
f_{x} & =\frac{1}{4 i \alpha} \operatorname{tr}\left(r_{x}\left[r_{x t}, r_{x x}\right]\right), \\
\phi_{1 x} & =-i \zeta\left(r_{3 x} \phi_{1}+r_{x}^{-} \phi_{2}\right), \\
\phi_{2 x} & =-i \zeta\left(r_{x}^{+} \phi_{1}-r_{3 x} \phi_{2}\right) .
\end{aligned}
$$

It is the M-XXXI equation written in the form of the equation with self-consistent sources.

\section{Conclusions}

In this paper, we have shown that the HFE equation admits an integrable generalization with the self-consistent sources - the M-XIV equation. In particular, the integrability of the M-XIV equation has been established by constructing its Lax pair. We have also demonstrated that the M-XIV equation is equivalent to the M-XXXII equation. At the level, some reductions of the M-XXXII equation are found. Another interesting issue is to generalize these results to the real and complex short pulse equations [104]. Although in this paper, we have restricted our consideration to the mathematical aspects of the proposed integrable two equations, the relevance of these equations as models capable of describing the dynamics of ultra-short pulses in optical fibers is an important issue to be studied in a future work. 


\section{Acknowledgements}

This work was supported by the Ministry of Edication and Science of Kazakhstan under grants 011800935 and 011800693.

\section{References}

[1] Sergyeyev A. New integrable $(3+1)$-dimensional systems and contact geometry, Lett. Math. Phys., 108, 359-376 (2018)

[2] Sergyeyev A. Integrable (3+1)-dimensional system with an algebraic Lax pair, arXiv:1812.02263

[3] Sergyeyev A. Integrable (3+1)-dimensional systems with rational Lax pairs, arXiv:1711.07395

[4] Szablikowski B.M., Blaszak M. Meromorphic Lax representations of (1+1)dimensional multi-Hamiltonian dispersionless systems, J. Math. Phys., 47, 092701 (2006)

[5] Blaszak M., Szablikowski B.M. From dispersionless to soliton systems via Weyl-Moyal like deformations, J. Phys. A: Math. Gen., 36, 12181 (2003)

[6] Blaszak M., Szablikowski B.M. Classical R-matrix theory of dispersionless systems: I. (1+1)-dimension theory, J. Phys. A: Math. Gen., 35, 10325 (2002)

[7] Bogdanov L.V., Pavlov M.V. Six-dimensional heavenly equation. Dressing scheme and the hierarchy, arXiv:1806.01500.

[8] Konno K., Oono H. New coupled dispersionless equation, J. Phys. Soc. Jpn., 63, 377-378 (1994)

[9] Kakuhata H., Konno K. A generalization of coupled integrable, dispersionless system, J. Phys. Soc. Jpn., 65, 340-341 (1996)

[10] Kotlyarov V.P. On equations gauge equivalent to the sine-Gordon and PohlmeyerLund-Regge equation, J. Phys. Soc. Jpn., 63, 3535-3537 (1994)

[11] V. K. Melnikov, Commun. Math. Phys. 120, 451 (1989);

[12] V. K. Melnikov, Commun. Math. Phys. 126, 201-215 (1989).

[13] V. K. Melnikov, Inverse Problem 6, 233-246 (1990).

[14] D. J. Kaup, Phys. Rev. Lett. 59, 2063 (1987). 
[15] J. Leon and A. Latifi, J. Phys. A 23, 1385 (1990).

[16] C. Claude, A. Latifi and J. Leon, J. Math. Phys. 32, 3321 (1991).

[17] R. A. Vlasov and E. V. Doktorov E V, Dokl. Akad. Nauk BSSR 26, 17 (1991).

[18] E. V. Doktorov and R. A. Vlasov, Opt. Acta 30, 223 (1993).

[19] M. Nakazawa, E. Yomada and H. Kubota, Phys. Rev. Lett. 66, 2625 (1991).

[20] E. V. Doktorov and V. S. Shchesnovich, Phys. Lett. A 207, 153 (1995).

[21] V. S. Shchesnovich and E. V. Doktorov, Phys. Lett. A 213, 23 (1996).

[22] V. K. Melnikov, J. Math. Phys. 31, 1106 (1990).

[23] J. Leon, J. Math. Phys. 29, 2012 (1988); Phys. Lett. A 144, 444 (1990).

[24] V. K. Melnikov, Phys. Lett. A, 133, 493 (1988).

[25] R. L. Lin, Y. B. Zeng and W. X. Ma, Solving the KdV hierarchy with self-consistent sources by inverse scattering method. Physica A, 291, 287-298 (2001)

[26] T. Schäfer and C. E. Wayne, Propagation of ultra-short optical pulses in cubic nonlinear media, Physica D 196, 90-105 (2004).

[27] Y. Chung, C.K.R.T. Jones, T. Schäfer and C.E. Wayne, Ultra-short pulses in linear and nonlinear media, Nonlinearity 18, 1351-1374 (2005).

[28] M. L. Rabelo, On equations which describe pseudospherical surfaces, Stud. Appl. Math. 81, 221-248 (1989).

[29] R. Beals, M. Rabelo and K. Tenenblat, Bäcklund transformation and inverse scattering solutions for some pseudospherical surface equations, Stud. Appl. Math. 81, 125-151 (1989).

[30] A. Sakovich and S. Sakovich, The short pulse equation is integrable, J. Phys. Soc. Jpn. 74, 239-241 (2005).

[31] J. C. Brunelli, The short pulse hierarchy, J. Math. Phys. 46, 123507 (2005).

[32] J.C. Brunelli, The bi-Hamiltonian structure of the short pulse equation, Phys. Lett. A 353, 475-478 (2006).

[33] A. Sakovich and S. Sakovich, Solitary wave solutions of the short pulse equation, J. Phys. A 39, L361-L367(2006). 
[34] Y. Matsuno, Multiloop soliton and multibreather solutions of the short pulse model equation, J. Phys. Soc. Jpn. 76, 084003 (2007).

[35] Y. Matsuno, Soliton and periodic solutions of the short pulse model equation, Handbook of Solitons: Research, Technology and Applications, edited by S. P. Lang and H. Bedore (Nova, New York, 2009) pp. 541-585.

[36] Y. Matsuno, A novel multi-component generalization of the short pulse equation and its multisoliton solutions, J. Math. Phys. 52, 123702 (2011).

[37] B.-F. Feng, An Integrable coupled short pulse equation, J. Phys A: Math. Theor. 45, $085202(2012)$.

[38] S. Sakovich, Transformation and integrability of a generalized short pulse equation, Commnu. Nonlinear Sci. Numer. Simulat. 39, 21-28 (2016).

[39] Y. Matsuno, Bilinear Transformation Method (Academic Press, New York, 1984).

[40] R. Hirota, The Direct Method in Soliton Theory (Cambridge University Press, 2004).

[41] R. Hirota and Y. Ohta, Hierarchy of coupled soliton equations. I, J. Phys. Soc. Jpn. 60, 798-809 (1991).

[42] A. Dimakis and F. Müller-Hoissen, Bidifferential calculas approach to AKNS hierarchies and their solutions, SIGMA 6, 055 (2010).

[43] B.-F. Feng, Complex short pulse and coupled complex short pulse equations, Physica D 297, 62-75 (2015).

[44] M. Wadati, H. Sanuki and K. Konno, Relationships among inverse method, Bäcklund transformation and an infinite number of conservation laws, Prog. Theor. Phys. 53, 419-436 (1975).

[45] D.V. Kartashov, A.V. Kim and S.A. Skobelov, Soliton structure of a wave field with an arbitrary number of oscillations in nonresonance media, JETP Lett. 78, 276-280 (2003).

[46] S.A. Skobelev, D.V. Kartashov and A.V. Kim, Few-optical-cycle solitons and pulse self-compression in a Kerr medium, Phys. Rev. Lett. 99, 203902 (2007).

[47] A.V. Kim, S.A. Skobelev, D. Anderson, T. Hansson and M. Lisak, Extreme nonlinear optics in a Kerr medium: Exact soliton solutions for a few cycles, Phys. Rev. A 77, 043823 (2008).

[48] Sh. Amiranashvili, A.G. Vladimirov and U. Bandelov, Solitary-wave solutions for few-cycle optical pulses, Phys. Rev. A 77, 063821 (2008). 
[49] S. Sakovich, Integrability of the vector short pulse equation, J. Phys. Soc. Jpn. 77, 123001 (2008).

[50] H. Leblond and D. Mihalache, Models of few optical cycle solitons beyond the slowly varying envelope approximation, Phys. Rep. 523, 61-126 (2013).

[51] Bao-Feng Feng. Complex short pulse and coupled complex short pulse equations. Physica D 297 (2015) 62-75. arXiv:1312.6431

[52] Feng B.-F., Maruno K., Ohta Y. Geometric formulation and multi-dark soliton solution to the defocusing complex short pulse equation. arXiv:1603.00781.

[53] Shen S., Feng B.-F. and Ohta Y. From the real and complex coupled dispersionless equations to the real and complex short pulse equations, Stud. Appl. Math., v.136, 6488 (2016).

[54] Matsuno Y. Integrable multi-component generalization of a modified short pulse equation. J. Math. Phys. 57, 111507 (2016). arXiv:1607.01079]

[55] Schafer T., Wayne C.E. Propagation of ultra-short optical pulses in cubic nonlinear media, Physics D, v.196, 90-105 (2004)

[56] G. Nugmanova, Z. Zhunussova, K. Yesmakhanova, G. Mamyrbekova, R. Myrzakulov. International Journal of Mathematical, Computational, Statistical, Natural and Physical Engineering, 9, N8, 328-331 (2015).

[57] J.-S. He, Y. Cheng, Y.-S. Li. Commun. Theor. Phys., 38, 493-496 (2002).

[58] U. Saleem, M. Hasan. J. Phys. A: Math. Theor., 43, 045204 (2010).

[59] M. Lakshmanan, Phil. Trans. R. Soc. A, 369 1280-1300 (2011).

[60] Chou K.S., Qu C.Z. Integrable equations arising from motions of plane curves, Physica, D, v.162, 9-33 (2002)

[61] M. Lakshmanan, Phys. Lett. A, 64, 53-54 (1977).

[62] Takhtajian L., Phys. Lett. A, 64, 53-54 (1977).

[63] R. Myrzakulov, S. Vijayalakshmi, G. Nugmanova, M. Lakshmanan Physics Letters A, 233 , 14-6, 391-396 (1997).

[64] R. Myrzakulov, S. Vijayalakshmi, R. Syzdykova, M. Lakshmanan, J. Math. Phys., 39, 2122-2139 (1998).

[65] R. Myrzakulov, M. Lakshmanan, S. Vijayalakshmi, A. Danlybaeva, J. Math. Phys., 39, 3765-3771 (1998). 
[66] Myrzakulov R, Danlybaeva A.K, Nugmanova G.N. Theoretical and Mathematical Physics, V.118, 13, P. 441-451 (1999).

[67] Myrzakulov R., Nugmanova G., Syzdykova R. Journal of Physics A: Mathematical \& Theoretical, V.31, 147, P.9535-9545 (1998).

[68] Myrzakulov R., Daniel M., Amuda R. Physica A., V.234, 13-4, P.715-724 (1997).

[69] Myrzakulov R., Makhankov V.G., Pashaev O.?. Letters in Mathematical Physics, V.16, N1, P.83-92 (1989)

[70] Myrzakulov R., Makhankov V.G., Makhankov A. Physica Scripta, V.35, N3, P. 233$237(1987)$

[71] Myrzakulov R., Pashaev O., Kholmurodov Kh. Physica Scripta, V.33, N4, P. 378-384 (1986)

[72] Anco S.C., Myrzakulov R. Journal of Geometry and Physics, v.60, 1576-1603 (2010)

[73] Myrzakulov R., Rahimov F.K., Myrzakul K., Serikbaev N.S. On the geometry of stationary Heisenberg ferromagnets. In: "Non-linear waves: Classical and Quantum Aspects", Kluwer Academic Publishers, Dordrecht, Netherlands, P. 543-549 (2004)

[74] Myrzakulov R., Serikbaev N.S., Myrzakul Kur., Rahimov F.K. On continuous limits of some generalized compressible Heisenberg spin chains. Journal of NATO Science Series II. Mathematics, Physics and Chemistry, V 153, P. 535-542 (2004)

[75] R.Myrzakulov, G. K. Mamyrbekova, G. N. Nugmanova, M. Lakshmanan. Symmetry, 7(3), 1352-1375 (2015). arXiv:1305.0098

[76] R.Myrzakulov, G. K. Mamyrbekova, G. N. Nugmanova, K. Yesmakhanova, M. Lakshmanan. Physics Letters A, 378, N30-31, 2118-2123 (2014). arXiv:1404.2088]

[77] Myrzakulov R., Martina L., Kozhamkulov T.A., Myrzakul Kur. Integrable Heisenberg ferromagnets and soliton geometry of curves and surfaces. In book: "Nonlinear Physics: Theory and Experiment. II". World Scientific, London, P. 248-253 (2003)

[78] Myrzakulov R. Integrability of the Gauss-Codazzi-Mainardi equation in 2+1 dimensions. In "Mathematical Problems of Nonlinear Dynamics", Proc. of the Int. Conf. "Progress in Nonlinear sciences", Nizhny Novgorod, Russia, July 2-6, 2001, V.1, P.314-319 (2001)

[79] Chen Chi, Zhou Zi-Xiang. Darboux Tranformation and Exact Solutions of the Myrzakulov-I Equations. Chin. Phys. Lett., 26, N8, 080504 (2009) 
[80] Chen Hai, Zhou Zi-Xiang. Darboux Transformation with a Double Spectral Parameter for the Myrzakulov-I Equation. Chin. Phys. Lett., 31, N12, 120504 (2014)

[81] Zhao-Wen Yan, Min-Ru Chen, Ke Wu, Wei-Zhong Zhao. J. Phys. Soc. Jpn., 81, 094006 (2012)

[82] Yan Zhao-Wen, Chen Min-Ru, Wu Ke, Zhao Wei-Zhong. Commun. Theor. Phys., 58, 463-468 (2012)

[83] K.R. Ysmakhanova, G.N. Nugmanova, Wei-Zhong Zhao, Ke Wu. Integrable inhomogeneous Lakshmanan-Myrzakulov equation, nlin/0604034

[84] Zhen-Huan Zhang, Ming Deng, Wei-Zhong Zhao, Ke Wu. On the integrable inhomogeneous Myrzakulov-I equation, [arXiv: nlin/0603069

[85] Martina L, Myrzakul Kur., Myrzakulov R, Soliani G. Journal of Mathematical Physics, V.42, 13, P.1397-1417 (2001).

[86] Xiao-Yu Wu, Bo Tian, Hui-Ling Zhen, Wen-Rong Sun and Ya Sun. Journal of Modern Optics, 2015.

[87] Z.S. Yersultanova, M. Zhassybayeva, K. Yesmakhanova, G. Nugmanova, R. Myrzakulov. International Journal of Geometric Methods in Modern Physics, 13, N1, 1550134 (2016).. arXiv:1404.2270

[88] Myrzakul Akbota and Myrzakulov Ratbay. Integrable Motion of Two Interacting Curves and Heisenberg Ferromagnetic Equations, Abstracts of XVIII-th Intern. Conference "Geometry, Integrability and Quantization", June 3-8, 2016, Bulgaria.

[89] Myrzakul Akbota and Myrzakulov Ratbay. Integrable motion of two interacting curves, spin systems and the Manakov system. International Journal of Geometric Methods in Modern Physics, 13, N1, 1550134 (2016). arXiv:1606.06598.

[90] Myrzakul Akbota and Myrzakulov Ratbay. Darboux transformations and exact soliton solutions of integrable coupled spin systems related with the Manakov system, arXiv:1607.08151.

[91] Myrzakul Akbota and Myrzakulov Ratbay. Integrable geometric flows of interacting curves/surfaces, multilayer spin systems and the vector nonlinear Schrodinger equation. International Journal of Geometric Methods in Modern Physics, 13, N1, 1550134 (2016). arXiv:1608.08553.

[92] Hussien R.A., Mohamed S.G. Generated Surfaces via Inextensible Flows of Curves in $R^{3}$. Journal of Applied Mathematics, v.2016, Article ID 6178961 (2016). 
[93] Myrzakulova Z., Myrzakul Akbota, Nugmanova G., Myrzakulov R. Notes on Integrable Motion of Two Interacting Curves and Two-layer Generalized Heisenberg Ferromagnet Equations. arXiv:1811.12216]

[94] Bekova G., Yesmakhanova K., Shaikhova G., NugmanovaG., Myrzakulov R. Integrable Flows of Curves/Surfaces, Generalized Heisenberg Ferromagnet Equation and Complex Coupled Dispersionless Equation. arXiv:1812.02152.

[95] Bekova G., Nugmanova G., Shaikhova G., Yesmakhanova K., Myrzakulov R. Geometric formulation and soliton solutions of the Myrzakulov-LXXIII equation and the complex short pulse equation,

[96] Bekova G., Nugmanova G., Shaikhova G., Yesmakhanova K., Myrzakulov R. Coupled Dispersionless and Generalized Heisenberg Ferromagnet Equations with SelfConsistent Sources: Geometry and Equivalence, arXiv:1901.01470]

[97] Hasimoto H. A soliton on a vortex filament, J. Fluid Mech., v.51, 477-485 (1972)

[98] Lamb G.L. Solitons and the motion of helical curves, Phys. Rev. Lett., v.37, 235-237 (1976)

[99] Lamb G.L. Solitons on moving space curves, J. Math. Phys., v.18, 1654-1661 (1977)

[100] Langer J., Perline R. Poisson geometry of the filament equation, J. Nonlinear Sci., v.1, 71-93 (1991)

[101] Langer J., Perline R. Curve motion inducing modified Korteweg - de Vries systems, Phys. Lett. A, v.239, 36-40 (1998)

[102] Goldstein R.E., Petrich D.M. The Korteweg - de Vries hierarchy as dynamics of closed curves in the plane, Phys. Rev. Lett. A, v.67, 3203-3206 (1991)

[103] Doliwa A., Santini P.M. An elementary geometric characterization of the integrable motion of a curves, Phys. Lett. A, v.185, 373-384 (1994)

[104] Myrzakulov R. et al. Real/Complex Short Pulse and Myrzakulov-XV equations with self-consistent sources, (2019)

[105] Valchev T. I., Yanovski A. B. New Reductions of a Matrix Generalized Heisenberg Ferromagnet Equation, arXiv:1802.03591.

[106] Gerdjikov V.S., Grahovski G.G., Mikhailov A.V., Valchev T.I. On Soliton Interactions for a Hierarchy of Generalized Heisenberg Ferromagnetic Models on $S U(3) / S(U(1) U(2))$ Symmetric Space, J. of Geometry and Symmetry in Physics, 25, 23-55 (2012). arXiv:1201.0534] 